\title{
Ossification of the Vascular Pedicle in Microsurgical Fibular Free Flap Reconstruction of the Head and Neck
}

\author{
C.M. Glastonbury, A. van Zante, and P.D. Knott
}

\begin{abstract}
BACKGROUND AND PURPOSE: The fibular free flap, often used for osseous reconstruction following extirpation of head and neck malignancies, has been associated with heterotopic periosteal ossification. We aimed to determine the frequency and radiologic characteristics of this process and describe its clinical correlates.
\end{abstract}

MATERIALS AND METHODS: Surgical records for 2 years and neck imaging reports for 10 years were evaluated to identify patients with fibular free flap reconstruction and CT and/or PET/CT imaging available for review. The images were evaluated for the quality, type, and contour of ossification, and the reports were reviewed for associated clinical findings and radiologic impressions.

RESULTS: Of 32 patients with posttreatment CT or PET/CT imaging, ossification was evident in 16 patients (50\%) as early as 1 month following fibular free flap reconstruction. In 8 patients, it mimicked a new bone; in 5, it appeared as linear attenuation; in 2, as multiple short segments; and in 1 patient, a mixed appearance was found. No associated FDG uptake was seen on PET/CT. On MR imaging, these findings were extremely subtle or not appreciable. In only 1 patient was new bone associated with symptoms.

CONCLUSIONS: Periosteal ossification of the vascular pedicle is commonly evident on CT following fibular free flap, even as early as 1 month after reconstruction, though the finding is not typically noted on imaging. While symptoms related to new bone are uncommon, they may mimic recurrent tumor. The location and pattern of ossification and the absence of a soft-tissue mass or FDG uptake are useful distinguishing imaging features.

ABBREVIATIONS: $\mathrm{FFF}=$ fibular free flap; $\mathrm{HNSCC}=$ head and neck squamous cell carcinoma

M icrosurgical free flaps are commonly used for reconstruction of surgical defects in patients with head and neck malignancies. Such flaps allow cosmetic and functional improvement following resection of neoplasms, and they may additionally provide protection for vulnerable tissues before radiation therapy. ${ }^{1,2}$ Following resection of all or part of the mandible or maxilla, osteocutaneous flaps may be used for reconstruction, with the most frequent choice being the fibular free flap (FFF). ${ }^{2}$ After fibula bone harvest, the distal aspect of the bone is osteotomized and contoured to fit the facial bone defect, while the vascular pedicle is carefully preserved and then anastomosed to available neck vessels in either the ipsilateral or contralateral neck. ${ }^{3}$

\footnotetext{
Received February 14, 2014; accepted after revision March 22.

From the Departments of Radiology and Biomedical Imaging (C.M.G.), Anatomic Pathology (A.v.Z.), and Otolaryngology-Head and Neck Surgery (C.M.G., P.D.K.), University of California, San Francisco, San Francisco, California.

Please address correspondence to C.M. Glastonbury, MD, Department of Radiology, Box 0628, Room L-358, 505 Parnassus Ave, San Francisco, CA 94143-0628;

e-mail: christine.glastonbury@ucsf.edu

三 Indicates article with supplemental on-line table.

http://dx.doi.org/10.3174/ajnr.A3979
}

Posttreatment imaging evaluation after free flap reconstruction is often complex, with loss of reliably symmetric anatomic landmarks and altered signal intensity (MR imaging) or attenuation (CT) of both native and flap tissues, particularly with denervation changes in the muscular component of myogenous flaps. Concurrently, the clinical examination after reconstruction can be difficult, both in the early postoperative edematous phase and the more delayed phase, especially when radiation is also delivered.

While the imaging features of microsurgical free flaps ${ }^{4-6}$ have been described and imaging features suggesting recurrent tumor have also been delineated, ${ }^{7,8}$ we have observed an unusual finding on neck CT scans of new bone developing in patients with prior fibular free flap placement. This new bone has been described in the surgical literature, predominantly in case reports, and has been ascribed to heterotopic ossification arising from fibula periosteum, which is preserved as part of the vascular pedicle during microvascular reconstruction. ${ }^{9-16}$ It has been described as a potential clinical pitfall, presenting as a new hard neck mass and mimicking recurrent disease. We sought to determine the frequency, timing, and radiologic characteristics of heterotopic 

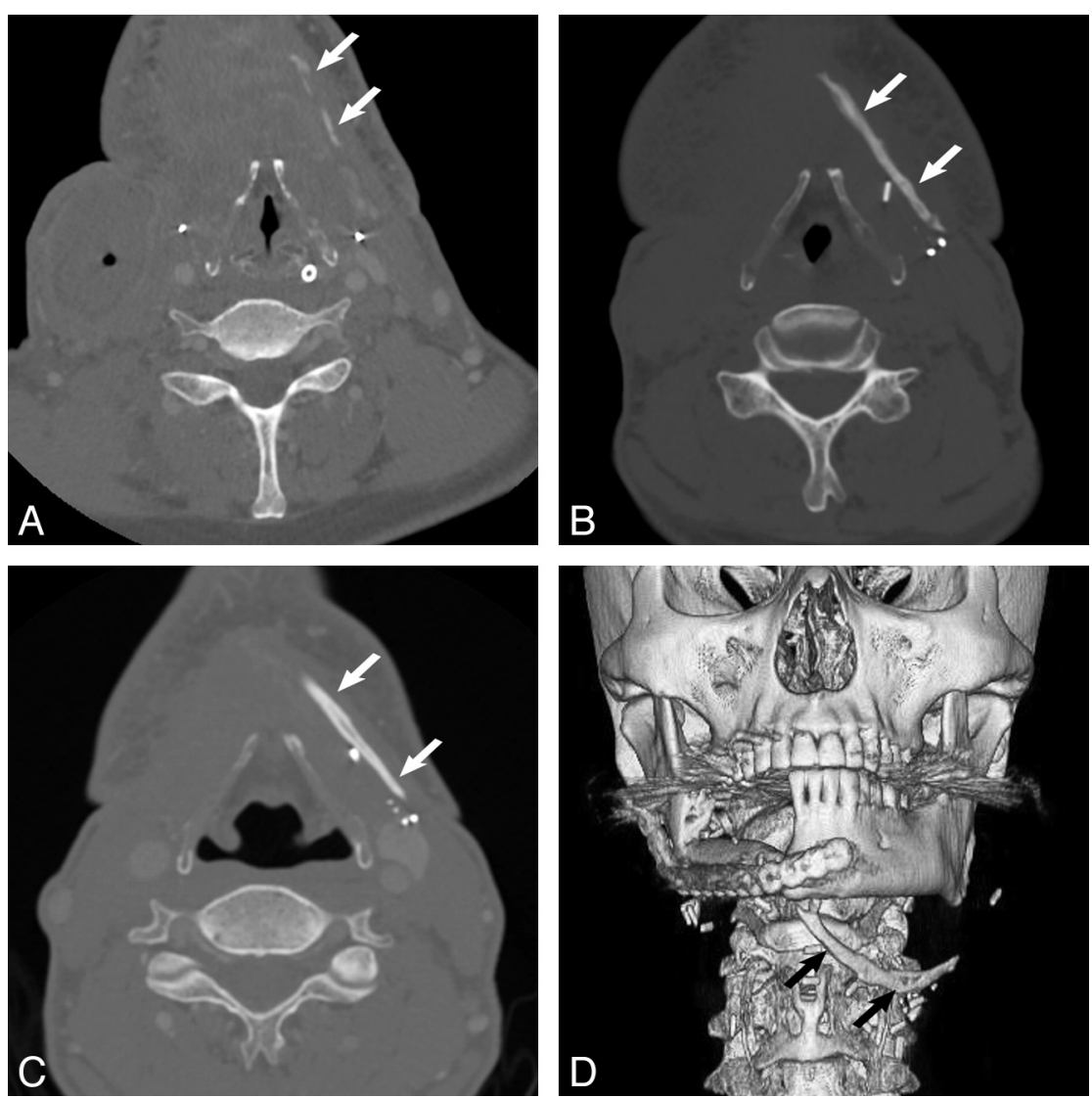

FIG 1. Axial contrast-enhanced neck CT scans and 3D reformat performed in a 59-year-old-man who underwent fibular free flap reconstruction for osteomyelitis complicating segmental mandibulectomy (patient 3). Imaging performed at 1 month (A), 2 months ( $B$ ), and 12 months $(C)$ shows progressive ossification in the left neck from linear segmental densities to a linear attenuation to a linear structure with features characteristic of bone (arrows). The new bone is of greater attenuation than enhancing vessels and is less attenuated than adjacent vascular clips. D, 3D reformation from a CT scan at 9 months when the pedicle ossification (arrows) was first mentioned in the imaging report.

ossification after flap placement and the frequency with which it was described in CT, MR imaging, and PET/CT imaging reports or was clinically concerning.

\section{MATERIALS AND METHODS}

The surgical records of a single head and neck reconstructive surgeon were evaluated to identify patients who had FFF reconstructive surgery and neck imaging available. In addition, all CT, PET/ $\mathrm{CT}$, and MR imaging radiology reports at our institution from July 2004 to July 2013 were searched for the terms "free flap," "flap reconstruction," "fibula flap," and "fibular flap" by using Illuminate (Softek, Version 2.0; http://www.softekinc.com/illuminate/) to identify imaging studies of patients with FFF neck reconstruction and imaging performed postoperatively. Institutional review board approval was obtained with consent waived for this retrospective evaluation of clinical notes and imaging records.

The inclusion criteria for the study were those patients with FFF reconstruction at our institution who had at least 1 postoperative $\mathrm{CT}$ or PET/CT study available for review. Any neck MR imaging examinations, if available for this cohort, were also reviewed.

The pertinent neck imaging examinations were reviewed by a Certificate of Added Qualification-certified neuroradiologist to determine the presence of ossification on CT, PET/CT, and/or
MR imaging. The images were evaluated for the size and type of ossification (linear or curved, continuous or multisegmented) and quality (dense or bonelike with cortical bone and marrow evident). If MR imaging was available, it was reviewed in a retrospective fashion to determine whether ossification could be identified. If multiple imaging studies were performed, these were reviewed sequentially to determine any changes in the development of ossification such as ongoing growth or resorption.

Evaluation of flaps by CT is performed as a postcontrast CT examination with 3-mm sections and sagittal and coronal reformations. Images are also reviewed on bone window algorithm images generated from the same data. All PET/CT at our institution for patients with head and neck pathology is routinely performed with intravenous contrast (unless contraindicated) and is reformatted with dedicated 3-mm neck CT sections. Neck MR imaging is performed on either a $1.5 \mathrm{~T}$ or $3 \mathrm{~T}$ magnet with 4-mm axial and coronal $\mathrm{T} 1$ and $\mathrm{T} 2$ fat saturated sequences and postcontrast axial and coronal T1 fat saturated sequences unless gadolinium is contraindicated.

The patient' clinical records were reviewed for the indication for FFF reconstruction, and the clinical notes were reviewed to determine whether concerning symptoms or a mass was evident clinically. The imaging reports were also reviewed to determine whether ossification had been reported.

\section{RESULTS}

\section{Patients and Imaging}

Using the search methods described, we identified 50 patients who had an FFF placed for facial bony reconstruction. Of this group, 14 were excluded because they had no postoperative imaging available, and 4 , because only postoperative neck MR imaging was available. Of the remaining 32 patients, 8 were women and 24 were men. They ranged in age from 29 to 78 years, with a mean age of 60.2 years. Eighteen patients had at least 1 posttreatment neck CT (with or without contrast) available for review, including 2 patients with only early postoperative scans at 1 and 3 weeks, respectively. Eighteen patients had at least 1 posttreatment PET/CT available for review, with 3 patients having both modalities performed. Of the group of 32 with CT of the neck and/or $\mathrm{PET} / \mathrm{CT}, 16$ also had neck MR imaging.

In 16 of the 32 patients (50\%), heterotopic periosteal ossification was identified. The new bone appeared as bone attenuation and was denser than intravascular iodinated contrast and less dense than metallic clips. The Table describes this study group and its findings. Of those with ossification, 2 were 

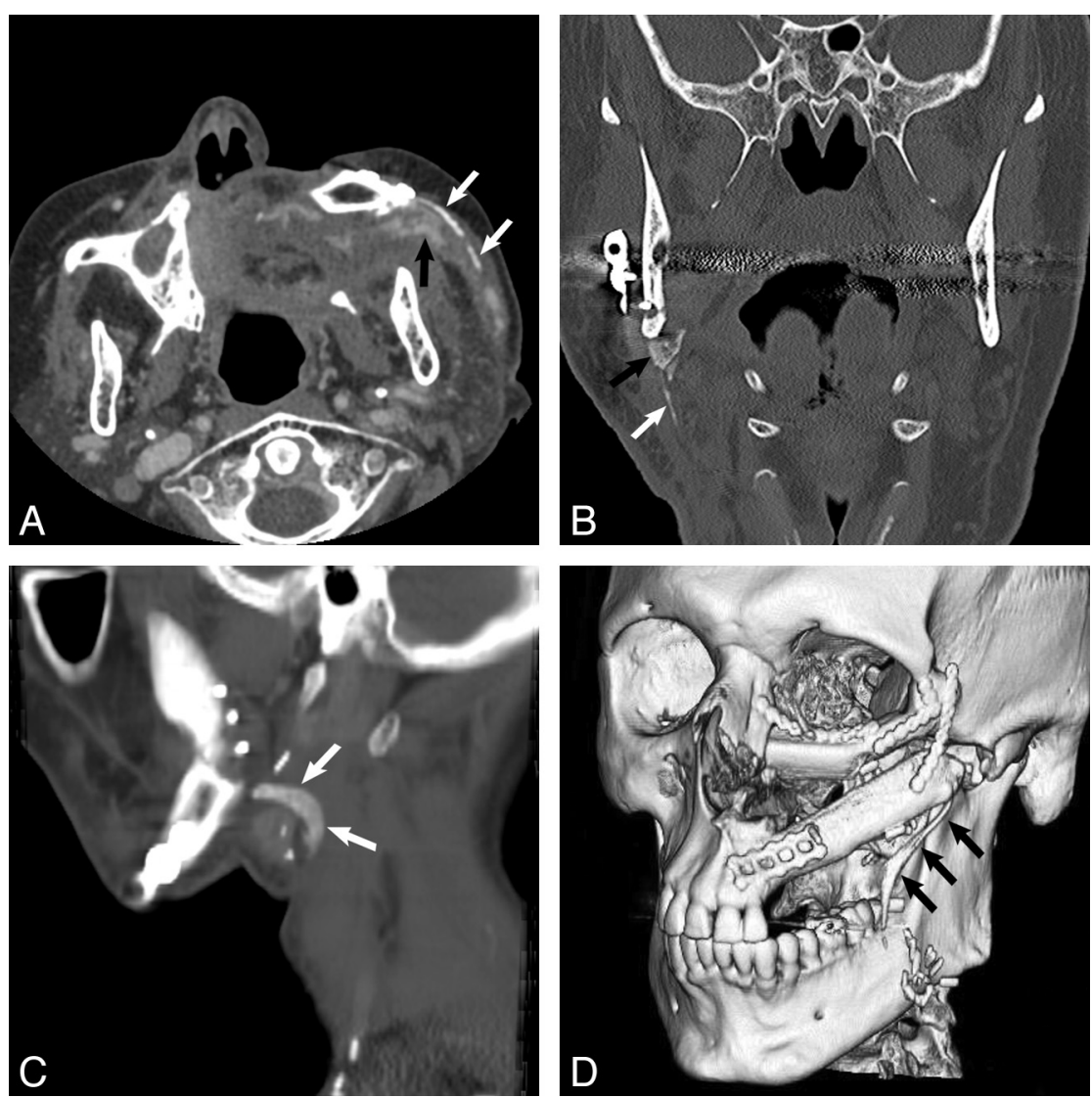

FIG 2. Different patterns of heterotopic ossification. Axial contrast-enhanced $C T(A)$ in a 77year-old man (patient 4) 5 months after FFF reconstruction shows multiple linear densities (white arrows); we classify this pattern as multisegmented ossification. Note that pedicle ossification is clearly denser than the adjacent enhancing flap vessels (black arrow). Coronal face CT (B) in a 72-year-old man (patient 9) 5 months after reconstruction shows vertical linear ossification (white arrow) with a more superior triangular bonelike component (black arrow). Sagittal reformat from neck CT (C) in a 51-year-old man (patient 6) 10 months after reconstruction for recurrent HNSCC shows curved bonelike ossification (arrows). 3D reformat (D) in a 37-year-old man (patient 2) 9 months after reconstruction following resection of a maxillary osteoblastic osteosarcoma shows a linear bonelike structure (arrows).

women and 14 were men. These patients ranged in age from 37 to 78 years (mean, 62.3 years) at the time of FFF reconstruction. Eight $(50 \%)$ had surgery for primary or recurrent head and neck squamous cell carcinoma (HNSCC); 2 (12.5\%) each, for failed prior reconstruction, metastatic disease, and osteoradionecrosis; 1 (6.25\%), for osteomyelitis; and 1, for osteosarcoma. Fourteen of $16(87.5 \%)$ cases were mandibular reconstructions, with the 2 maxillary reconstructions performed for osteoblastic osteosarcoma and failed previous flap after subtotal maxillectomy, respectively.

\section{Patterns of Ossification}

In 8 patients $(50 \%)$, pedicle ossification mimicked development of a "new bone" with cortical and marrow densities evident on CT (Figs 1 and 2). Five of these were linear bonelike structures, 2 of 8 bonelike densities were curved, and 1 of 8 was a rectangular segment of bone. In 5 patients (31\%), the ossification appeared as long dense linear segments, and in 2 patients (13\%), as multiple short segments of linear density. One patient had a mixed appearance with a long segment of linear density and a short triangular bonelike component. For the bonelike components, the aver- age thickness and length were $3 \mathrm{~mm}$ and $3.7 \mathrm{~cm}$, respectively. For attenuated ossification components, the average thickness and length were $2.5 \mathrm{~mm}$ and $2.7 \mathrm{~cm}$, respectively.

In the 6 patients with ossification and multiple CT or PET/CT scans, the ossification was seen to thicken and progress and/or stabilize; it was not seen to resorb or resolve in any case, with the maximum time to imaging of 20 months postreconstruction. The earliest imaging findings were noted on CT or PET/CT scans obtained at 1 month in 3 patients who all had subsequent confirmatory imaging scans. All patients who had ossification had evidence of it on their earliest available CT or PET/CT, and 2 of the linear bonelike structures began as linear densities on the earliest scan.

None of the 16 ossification cases showed a soft-tissue mass in association with the new bone and none of the 8 cases with PET/CT showed FDG uptake above that of background activity associated with the bone. In the 9 ossification cases that also had MR imaging, it was extremely difficult to confidently identify the ossification, even in reviewing the scans alongside the CT scans with abnormal findings and even in cases with thicker bonelike growths.

One patient from the original group of 32 was only imaged at 3 weeks postoperatively and showed no evidence of ossification, but dense tram-track-like vascular calcification of the peroneal artery was present, which was clearly identifiable and distinguishable from the ossification seen in other cases (Fig 3).

\section{Radiology and Clinical Reports}

On review of the CT imaging reports, the presence of new bone or new density was mentioned in only 1 of the patients. The possibility of a retained foreign body was raised in a neck CT scan obtained at 9 months after FFF because the attenuated structure was retrospectively noted to be present 1 month postreconstruction (Fig 1). Clinical examination of this patient revealed a firm mass in the neck that followed the course of the vascular pedicle. The patient subsequently underwent neck surgery for an unrelated issue, and this tissue was resected, demonstrating well-organized lamellar bone (Fig 4). This was the only patient with a clinical neck mass or reported symptoms referable to the ossification.

\section{DISCUSSION}

A fibular free flap is a vascularized osteocutaneous flap used for reconstruction of composite surgical defects of the head and neck. In our practice, it is most often used for reconstruction following segmental mandibulectomy or hemimandibulectomy for squa- 
mous cell carcinoma, but it may also be used after resection of other head and neck, jaw, or facial neoplasms and for reconstruction following jaw osteoradionecrosis. The flap can provide up to $25 \mathrm{~cm}$ of cortical bone in addition to cutaneous cover and is adequate bone for osseointegrated (dental) implants, which are usually placed later in a second-stage surgery. The bone is fixed in place with reconstruction plates while microvascular anastomosis is performed. ${ }^{1-3}$

We found 16 cases of ossification of the vascular pedicle among 32 patients (50\%) with FFF and at least 1 posttreatment CT or PET/CT scan, suggesting that this is not an uncommon finding. While flap ossification is described as rare in the literature, it has also been variably reported as occurring in 17\%-65\% of neck CT scans and on orofacial panoramic films in $27 \%$ of cases in different surgical studies. ${ }^{9,11,16}$ One craniofacial article reported more frequent occurrence of heterotopic ossification in FFF reconstructions performed for maxilla resections. ${ }^{12}$ Multiple theories have been proposed to account for this new bone formation, including active fracture repair at the osteotomy site, growth factors, mechanical tension, inflammation, and increased blood flow allowing osteoprogenitor recruitment. ${ }^{12,14,16}$ These factors

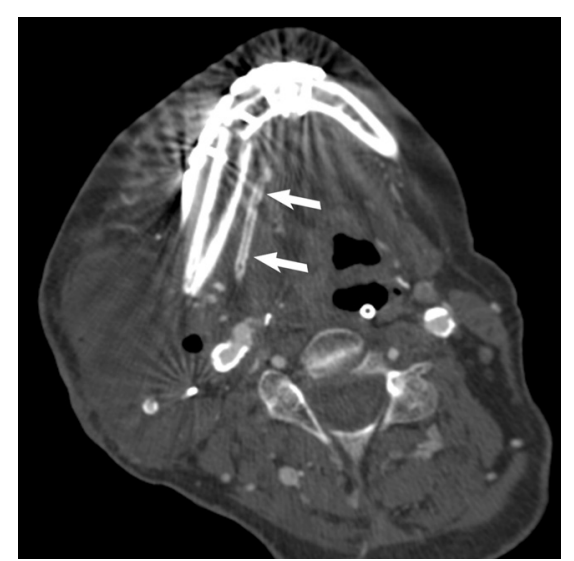

FIG 3. Axial nonenhanced CT in a 73-year-old man 3 weeks after FFF reconstruction shows tram-track vascular calcification in the wall of the peroneal artery (arrows), a pattern typical of atherosclerosis. This appears distinctly different from the attenuated, linear, continuous, or multisegmented patterns that were observed in patients with periosteal ossification. Pedicle ossification is not evident on this scan, and the patient had no further CT or PET/CT follow-up at our institution.
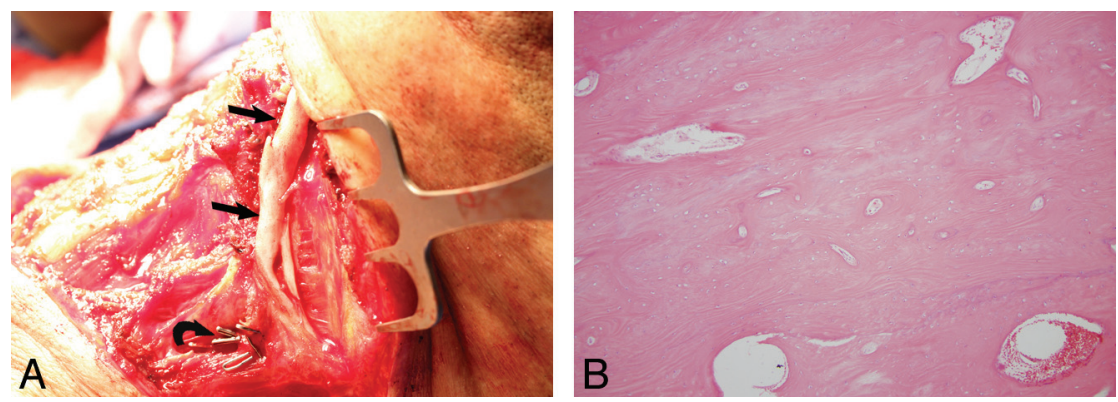

FIG 4. Operative and histopathology images from same patient as in Fig 1 (patient 3). Intraoperative photograph $(A)$ at 13 months following fibula microvascular transfer demonstrates pronounced heterotopic calcification of the fibula periosteum (arrows). Also note surgical clips (curved arrow) that were placed on side branches during the fibula transfer. Histologic section of this resected bone $(B)$ with hematoxylin and eosin stain at magnification of 200X shows mature lamellar bone without marrow elements. This bone is microscopically indistinguishable from native bone, such as the hyoid. may all influence the development of bone formation, but the key underlying characteristic of the FFF is the presence of periosteum from the fibula to which the peroneal vascular pedicle is attached.

During harvest of the fibula, virtually the entire bone is taken (up to $25 \mathrm{~cm}$ ), while only the distal one-half or one-third of the bone is typically used for reconstruction of the facial bone defect. The proximal one-half to two-thirds of the fibular bone is discarded; however, the contiguous periosteum of the discarded proximal bone is preserved because the vascular pedicle is intimately associated with it and cannot be easily dissected from it. In cases in which the pedicle must reach distant recipient neck vessels, such as in the contralateral neck or to the transverse cervical vessels, the osseous component of the flap may be short and the pedicle with the overlying attached periosteum may be relatively long. This segment of fibular periosteum remains with the FFF and is implanted in the neck, either along the native bone or over the vessels to protect the anastomosis. A recent surgical study had a prospective arm in which 20 patients had a modified harvesting procedure with removal of this fibular periosteum before vascular anastomosis, and none of these patients developed flap ossification in the 18 months following FFF reconstruction. ${ }^{16}$ Radiation therapy has also been described as a potential factor in the induction of periosteal ossification but does not appear to influence the onset or extent of flap ossification in either the literature or this current study. ${ }^{12}$

Of the group of 32 patients with FFF and imaging available for review, 2 of 8 women (25\%) and 14 of 24 men (58\%) developed ossification. The literature does not report a similar sex bias for the occurrence of ossification, and with our small numbers, we are unsure of the reason for this more common frequency in men. While hormones have been proposed as potentially having a role, ossification has been reported in males and females in wide age ranges from pubescence to adults, including postmenopausal women, as is the case with our 2 patients. ${ }^{9-13,16}$

Reported clinical manifestations of pedicle ossification include trismus, severe pain with mastication or head turning, submandibular swelling, or a hard palpable mass, though it is evident in the literature that such clinical findings are present in less than one-third of patients with imaging evidence of ossification and only 1 of our 16 patients was symptomatic. ${ }^{10,14-16}$ These clinical symptoms may mimic or raise clinical concern for recurrent tumor. The development of ossification in our patients was all within the 2-year postreconstruction period, when the concern for local tumor recurrence in HNSCC cases is greatest.

In symptomatic cases, the ossified pedicle and its surrounding periosteal tissues may be excised. In asymptomatic cases, there appears to be no surgical indication for resection. That heterotopic ossification of the vascular pedicle is largely asymptomatic undoubtedly accounts for its rare description in the literature. In all except 1 of our patients, 
flap ossification was not noted in the radiology report, even when attaining several centimeters in size. The radiologist noting the possibility of a foreign body suggests that radiologists may also not be aware of this phenomenon. The attenuated linear pattern of early new bone may be mistaken for vascular clips, though these are distinctly denser than bone. Mature bone is also less attenuated than contrast-enhancing flap vasculature and appears distinctly different from the tram-track pattern of vascular calcification.

We were only able to identify pedicle ossification on neck MR imaging with careful retrospective review, suggesting that CT (or PET/CT) may be of greater value than MR imaging when a hard mass develops in patients with prior FFF reconstruction.

This retrospective study is limited in that fibular free flap reconstruction at our institution is not routinely followed by CT or PET/CT. In addition, follow-up imaging studies were not always performed at our institution and/or available for review or performed in a defined timeframe. Thus, it is not possible to determine the exact frequency or timing of heterotopic ossification in this setting, though our results agree with the current surgical literature and suggest that it is a reasonably common and stable finding, evident even in the early months following reconstruction.

\section{CONCLUSIONS}

Ossification of the vascular pedicle arising from the periosteum associated with a fibular free flap reconstruction is a reasonably common finding on CT examinations of the neck as early as 1 month following reconstruction. This finding is not associated with FDG avidity on PET/CT examinations and is typically very subtle or imperceptible on MR imaging. It may uncommonly present with trismus, pain, or a palpable mass and can be readily differentiated on CT from peroneal artery atherosclerosis, retained surgical material, and, perhaps more important, from recurrent disease by the surgical history, the contour and attenuation of the ossification, and the lack of an associated soft-tissue mass.

Disclosures: Christine M. Glastonbury-UNRELATED: provided consultancy to and received royalties and stocks/stock options from Amirsys. P. Daniel KnottUNRELATED: member of the Genentech Basal Cell Carcinoma Surgical Advisory Board. This board meets 1-2 times per year. In no way is there any conflict of interest or any relevance of this relationship to the submitted research.

\section{REFERENCES}

1. Wong $\mathrm{CH}$, Wei FC. Microsurgical free flap in head and neck reconstruction. Head Neck 2010;32:1236-45

2. Hayden RE, Mullin DP, Patel AK. Reconstruction of the segmental mandibular defect: current state of the art. Curr Opin Otolaryngol Head Neck Surg 2012;20:231-36

3. Wehage IC, Fansa H. Complex reconstructions in head and neck cancer surgery: decision making. Head Neck Oncol 2011;3:14

4. Learned KO, Malloy KM, Loevner LA. Myocutaneous flaps and other vascularized grafts in head and neck reconstruction for cancer treatment. Magn Reson Imaging Clin N Am 2012;20:495-513

5. Hudgins PA. Flap reconstruction in the head and neck: expected appearance, complications, and recurrent disease. Eur J Radiol 2002;44:130-38

6. Chong J, Chan LL, Langstein HN, et al. MR imaging of the muscular component of myocutaneous flaps in the head and neck. AJNR Am J Neuroradiol 2001;22:170-74

7. Hudgins PA, Burson JG, Gussack GS, et al. CT and MR appearance of recurrent malignant head and neck neoplasms after resection and flap reconstruction. AJNR Am J Neuroradiol 1994;15:1689-94

8. Tomura N, Watanabe $\mathrm{O}$, Hirano $\mathrm{Y}$, et al. MR imaging of recurrent head and neck tumours following flap reconstructive surgery. Clin Radiol 2002;57:109-13

9. DeConde AS, Vira D, Blackwell KE, et al. Neck mass due to pedicle ossification after oromandibular reconstruction. Laryngoscope 2011;121:2095-99

10. Smith RB, Funk GF. Severe trismus secondary to periosteal osteogenesis after fibula free flap maxillary reconstruction. Head Neck 2003;25:406-11

11. Karagozoglu KH, Winters HA, Forouzanfar T, et al. Periosteal ossification of the vascular pedicle after reconstruction of continuity defects of the mandible and the maxilla with fibular free flaps: a retrospective study. Br J Oral Maxillofac Surg 2013;51: 965-67

12. Myon L, Ferri J, Genty M, et al. Consequences of bony free flap's pedicle calcification after jaw reconstruction. J Craniofac Surg 2012;23:872-77

13. Acartürk TO, Aslaner EE. Periosteal ossification from the vascular pedicle of a free fibular flap. J Craniofac Surg 2011;22:e29-32

14. González-García R, Manzano D, Ruiz-Laza L, et al. The rare phenomenon of vascular pedicle ossification of free fibular flap in mandibular reconstruction. J Craniomaxillofac Surg 2011;39:114-18

15. Autelitano L, Colletti G, Bazzacchi R, et al. Ossification of vascular pedicle in fibular free flaps: a report of four cases. Int J Oral Maxillofac Surg 2008;37:669-71

16. Tarsitano A, Sgarzani R, Betti E, et al. Vascular pedicle ossification of free fibular flap: is it a rare phenomenon? Is it possible to avoid this risk? Acta Otorhinolaryngol Ital 2013;33:307-10 